\title{
Rare case of recurrent stroke in a patient with eosinophilic granulomatosis with polyangiitis: a case report
}

\author{
Mirjana Arsenijević ${ }^{1 \dagger}$, Nikola Ivančević ${ }^{\dagger}$, Dejana Jovanović $c^{1,3}$, Milan Radović ${ }^{3,4}$ and Ivana Berisavac ${ }^{1,3^{*}}$
}

\begin{abstract}
Background: Central nervous system involvement is rarely described in eosinophilic granulomatosis with polyangiitis (EGPA) and occurs in 5-9\% of patients. Among central nervous system manifestations, cerebral infarctions are the most common. To the best of our knowledge, a recurrent stroke in patients with EGPA without cardiac risk factors during maintenance therapy so far has not been described.

Case presentation: A previously healthy 57-year-old female during the course of 1 year developed asthma, sinusitis, polyneuropathy, muscle weakness, and rash followed by fatigue, myalgia, arthralgia, and fever. After an initial diagnostic evaluation, elevated values of eosinophils, liver enzymes, creatine kinase, lactate dehydrogenase, and inflammatory markers (sedimentation rate and C-reactive protein) were found, and renal impairment was detected. On the third day of hospitalization, she developed left-sided hemiparesis due to an ischemic stroke in the right basal ganglia. She has been diagnosed with EGPA, and oral corticosteroid, immunosuppressive, and antiplatelet therapy were applied. Despite potent treatment and initial recovery, a few weeks later, she developed recurrent ischemic stroke in the left hemisphere and pulmonary embolism as rare and potentially severe complications of EGPA. Assuming that complete disease remission had not been established previously, oral prednisone was initially substituted with intravenous methylprednisolone pulses. During follow-up, immunosuppressive therapy was slowly discontinued, oral corticosteroid therapy was reduced to a maintenance dose, and thromboembolic events were well controlled by oral anticoagulant therapy.

Conclusion: Anticoagulant therapy, in addition to immunosuppressive maintenance therapy, should be considered in any EGPA patient who has had an ischemic stroke even without cardiac risk factors.
\end{abstract}

Keywords: Eosinophilic granulomatosis with polyangiitis, Vasculitis, Rare complication, Recurrent stroke

\section{Background}

Eosinophilic granulomatosis with polyangiitis (EGPA), also called Churg-Strauss syndrome, is a rare systemic necrotizing vasculitis which affects the small- to medium-sized blood vessels and belongs to the group of antineutrophil cytoplasmic antibody (ANCA)-associated

\footnotetext{
* Correspondence: ivanaberisavac@gmail.com

${ }^{\dagger}$ Mirjana Arsenijević and Nikola Ivančević contributed equally to this work.

'Neurology Clinic, University Clinical Center of Serbia, Dr. Subotic Street 6,

Belgrade 11000, Serbia

${ }^{3}$ Medical Faculty, University of Belgrade, Dr. Subotic Street 8, Belgrade 11000,

Serbia

Full list of author information is available at the end of the article
}

vasculitides [1]. Its annual incidence ranges from 0.5 to 6.8 new cases per million inhabitants [2]. EGPA is associated with asthma and eosinophilia and typically involves the upper and lower respiratory tracts, lungs, nervous system, skin, heart, and kidneys [1].

Peripheral nervous system involvement is frequent with a prevalence of $55-72 \%$, while central nervous system (CNS) involvement is rarely described and occurs in 5-9\% of patients with EGPA [1]. Although rare, CNS involvement has been recognized as a poor prognostic factor, which is why it was included in the first tool designed to assess the prognosis at diagnosis [3]. Among 
CNS manifestations, cerebral infarct and subarachnoid hemorrhage are most often described [4]. To the best of our knowledge, this is the first case to report a recurrent stroke in patients with EGPA without cardiac risk factors during maintenance therapy.

\section{Case presentation}

Our patient is a 57-year-old woman. She had been completely healthy until 1 year before hospitalization, when she was diagnosed with asthma and chronic sinusitis. Progressive discomfort and tingling in the soles and later in the palms appeared 3 months before admission. Later, pain and mild weakness in the feet and hands emerged, and a rash on the legs occurred. Two weeks before hospitalization, she noticed worsening of asthma and sinusitis, worsening of painful sensations on the soles and palms, malaise, languor, pain in the muscles and joints, and gait disturbances due to previous symptoms.

On admission, she complained of malaise and fatigue, myalgia, and arthralgia. She had a body temperature of $37.5^{\circ} \mathrm{C}$, serous nasal discharge, and a purpuric rash on her legs. Muscle strength assessed using the Medical Research Council (MRC) rating scale was reduced globally (proximal upper limbs, MRC grade 4/5; proximal lower limbs, 3/5; distal upper limbs, 4/5; distal lower limbs, 3/ 5). An examination of her sensory systems showed paresthesia, dysesthesia, and allodynia in her soles and palms. In the initial laboratory, the absolute number of eosinophils was $9.95 \times 10^{9} / \mathrm{L}$, and they made up $50 \%$ of white blood cells. Creatine kinase, lactate dehydrogenase, aspartate aminotransferase, and alanine aminotransferase (2901 IU/L, $1137 \mathrm{IU} / \mathrm{L}, 97 \mathrm{IU} / \mathrm{L}$, and $137 \mathrm{IU} / \mathrm{L}$, respectively), as well as erythrocyte sedimentation rate and Creactive protein, were elevated.

On the third hospital day, she presented with left-sided hemiparesis. The first brain computer tomography $(\mathrm{CT})$ had been normal, but control brain CT performed $48 \mathrm{~h}$ after symptom onset showed a subacute ischemic lesion in the right basal ganglia region (Fig. 1a). Head magnetic resonance imaging (MRI) confirmed this finding (Fig. 1b, c). Doppler ultrasonography of the neck arteries and transcranial Doppler were normal. Cardiological evaluation, troponin level, electrocardiogram, $24 \mathrm{~h}$ electrocardiogram, and cardiac ultrasound were also normal.

Coagulation status was in the reference range. Studies of inherited (factor V Leiden, prothrombin G20210A, protein $\mathrm{C}$ and $\mathrm{S}$ deficiency, antithrombin deficiency, methylenetetrahydrofolate reductase/MTHFR C677T) and acquired (lupus anticoagulant, IgM and IgG anticardiolipin, and anti- $\beta 2$ glycoprotein 1 antibodies) thrombophilia were negative. Electromyoneurography showed the presence of myopathy and sensorimotor, axonal polyneuropathy in the upper and lower extremities. Metabolic and paraneoplastic causes of polyneuropathy were excluded. Hematological and infectious (Trichinella spiralis, Toxoplasma gondi, Toxocara canis, Brucella spp., Echinococcus, Cysticercus, and other helminthic infections, HIV, etc.) causes of eosinophilia also were excluded. The immunological analysis revealed elevated pANCA antibodies. Estimated urinary protein excretion was $2.3 \mathrm{~g} /$ day at admission (serum creatinine level 47 $\mu \mathrm{mol} / \mathrm{L}$; urine sediment was normal). CT of the thorax showed a normal finding.

Based on the presence of asthma, sinusitis, polyneuropathy, hypereosinophilia, and positive pANCA antibodies, EGPA was diagnosed. Intravenous corticosteroid therapy with methylprednisolone (starting $1000 \mathrm{mg} /$ day for 5 days followed by reduced doses) was administered for 2 weeks. After the third dose of intravenous methylprednisolone, the absolute number of eosinophils was $0.55 \times 10^{9} / \mathrm{L}$ (7.8\% of white blood cells), and the estimated urinary protein excretion was $1.3 \mathrm{~g} /$ day. Treatment was continued with oral prednisone therapy $(1 \mathrm{mg} /$ $\mathrm{kg} /$ day $)$ in combination with oral azathioprine $(2 \mathrm{mg} / \mathrm{kg} /$ day). During hospitalization, improvement of physical and neurological status and further normalization of blood and urine findings were noted. After 45 days of hospitalization, she was discharged with mild left-sided hemiparesis but was able to walk without assistance. Her therapy on discharge was prednisone $(1 \mathrm{mg} / \mathrm{kg} /$ day in tapering doses), azathioprine ( $2 \mathrm{mg} / \mathrm{kg} /$ day), and acetylsalicylic acid (100 mg/day).

Forty days later, during the outpatient control, global weakness of the right extremities and dyspnea were noticed. Due to clinical deterioration, she was hospitalized again. CT of the brain showed a subacute ischemic lesion in the left region of the basal ganglia (Fig. 1d) and a chronic ischemic lesion in the region of the basal ganglia on the right side (documented earlier, Fig. 1a). Control eosinophils, CRP, and $24 \mathrm{~h}$ proteinuria were in the normal range. Elevated D-dimer value of $9.87 \mathrm{mg} / \mathrm{L}$ (cutoff value $<0.5 \mathrm{mg} / \mathrm{L}$ ) was observed, while the rest of the coagulation status was in the normal range. Control analyses of lupus anticoagulant, IgM and IgG anticardiolipin, and anti- $\beta 2$ glycoprotein 1 antibodies were negative, while the followup level of pANCA was decreased. Thorax CT revealed segmental pulmonary embolism in the right lower lobe. Cardioembolism, atrial or interventricular septal defect, and deep vein thrombosis were ruled out by ultrasound of the heart and peripheral veins. Subcutaneous lowmolecular-weight heparin (enoxaparin sodium at dose 6000 units every $12 \mathrm{~h}$ ) was administered. Assuming that complete remission of the disease had not been achieved previously, intravenous methylprednisolone therapy (1000 $\mathrm{mg}$ /day) was administered for 5 days, followed by oral prednisone $(1 \mathrm{mg} / \mathrm{kg} /$ day $)$ which was gradually reduced, oral coumarin anticoagulant therapy was introduced, and pregabalin was given for neuropathic pain. The patient 

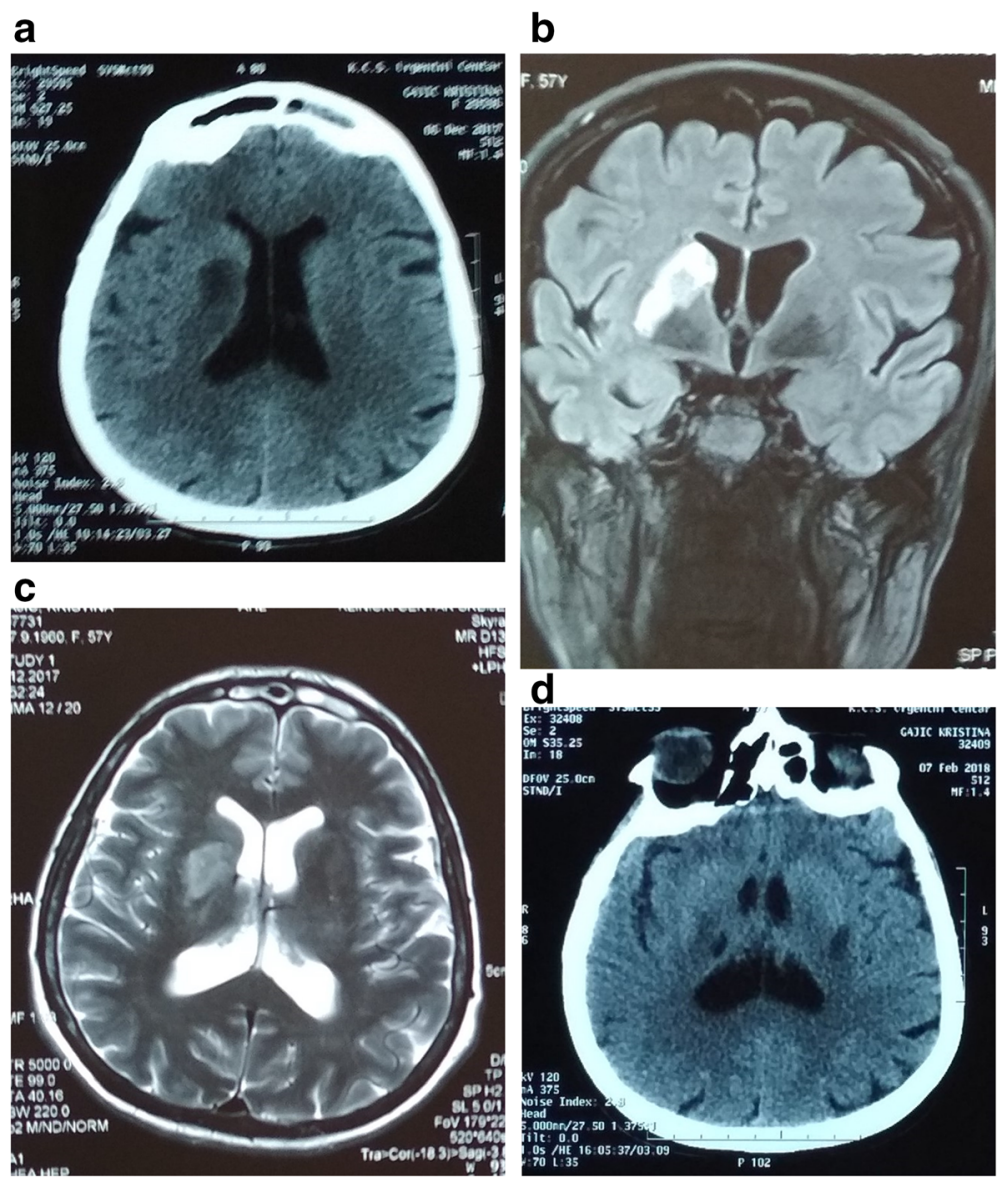

Fig. 1 a Head CT-the arrow points the sub-acute ischemic stroke in the basal ganglia region on the right. $\mathbf{b}$ Head MRI/FLAIR coronal plane section - the arrow points the sub-acute ischemic stroke in the basal ganglia on the right. $\mathbf{c}$ Head MRI/T2W transversal plane section - the arrow points the sub-acute ischemic stroke in the basal ganglia on the right. $\mathbf{d}$ Control head $\mathrm{CT}$ - the arrow points the new sub-acute ischemic stroke in the basal ganglia on the left; the arrowhead points the previous ischemic stroke sequel on the right (shown in a). CT, computer tomography; MRI, magnetic resonance imaging

has entered stable remission. During the 3-year follow-up, control neurological findings have shown the maintenance of mild left side hemiparesis accompanied by action left foot dystonia, oral prednisone has been reduced to a maintenance dose of $10 \mathrm{mg} /$ day, and azathioprine therapy has been slowly discontinued, while neuropathic pain has been well controlled.

\section{Discussion}

Our patient initially had positive four out of six diagnostic criteria for EGPA: asthma, sinusitis, eosinophilia, and polyneuropathy [5]. ANCA positivity, malaise and fatigue, fever, myositis, skin changes, kidney involvement, pulmonary embolism, and CNS involvement seen in our subject are also reported in EGPA [1, 4, 6, 7]. As already mentioned, CNS manifestations in EGPA are rare, but when they occur, ischemic strokes are the most common among them [4]. In some cases, abrupt onset, monophasic course, and multiple brain lesions indicate possible cardioembolism due to endomyocarditis in EGPA [8]. Other possible causes of stroke could be small vessel CNS arteritis, hypercoagulable state, patent foramen ovale (PFO), and heterozygotic factor V Leiden mutation $[4,8]$. Our patient did not have cardioembolism, PFO, coagulation disorder, or vasculitis of other etiologies. The initial stroke could be due to endothelial dysfunction and hypercoagulable state caused by hypereosinophilia $[4,8]$. Immunological analyses ruled out antiphospholipid syndrome in both cerebrovascular events. Therapy was administered according to the Consensus Task Force recommendations [6]. According to these recommendations, glucocorticoids are the cornerstone of therapy for EGPA and in patients with life-threatening symptoms, including CNS manifestations, and treatment should be initiated with methylprednisolone pulses [6]. In our case, the initial clinical response to corticosteroids was excellent and evident after the third dose (decrement in eosinophilia from 50 to $7.8 \%$ and urine protein excretion from 2.3 to $1.3 \mathrm{~g} /$ 
day). Taking into account this good response on glucocorticoids, the presence of the life-threatening event, and the severe side effects of cyclophosphamide, in the later course, we decided on maintenance therapy with oral prednisone and oral azathioprine [6,9]. As there are no official recommendations for secondary stroke prophylaxis in patients with EGPA, we have introduced an antiplatelet drug.

The appearance of new symptoms (recurrent stroke and pulmonary embolism) could reflect the activity of the disease despite the absence of correlation with laboratory findings (eosinophil count), because maintenance therapy (primarily prednisone) affects these laboratory values $[6,9,10]$. The potential explanation for this combined arterial and venous thromboembolism in the absence of cardiac risk factors and antiphospholipid syndrome could be the shift of the endothelium from an antiadhesive and antithrombotic state to a proadhesive and prothrombotic state in the presence of pANCA and/or through the release of specific eosinophil cytokines which could be expected due to decreased of eosinophils by previous therapy [11]. Due to the appearance of new life-threatening events, the patient had been re-treated with methylprednisolone pulses followed by oral prednisone, while azathioprine therapy had been initially continued, and after remission has been established, it was gradually stopped $[6,9]$. According to the recommendations, anticoagulant therapy has been introduced to prevent new thromboembolic events $[6,9]$. After this treatment correction, the patient has entered stable remission.

\section{Conclusion}

Our case suggests that an individually tailored therapeutic approach is necessary for each patient with EGPA and that even in patients with ischemic stroke without cardiac risk factors, anticoagulant therapy should be considered in addition to maintenance therapy.

\section{Abbreviations \\ EGPA: Eosinophilic granulomatosis with polyangiitis; ANCA: Antineutrophil cytoplasmic antibody; CNS: Central nervous system; MRC: Medical Research Council; CT: Computer tomography; MRI: Magnetic resonance imaging; MTHFR: Methylenetetrahydrofolate reductase; PFO: Patent foramen ovale}

\section{Acknowledgements}

None.

\section{Authors' contributions}

All authors contributed to the preparation and writing of this case presentation. Material preparation, data collection, and analysis were performed by MA, NI, DRJ, MR, and IB. The first draft of the manuscript was written by MA and $\mathrm{NI}$, and all authors have commented on the previous versions of the manuscript. The whole research was conceptualized and supervised by IB. All authors have read and approved the final manuscript.

\section{Funding}

None.

Availability of data and materials

Additional data that support the findings of this study are available on request from the corresponding author.

\section{Declarations}

Ethics approval and consent to participate

Not applicable.

\section{Consent for publication}

Written informed consent was obtained from the patient to publish case details and pictures.

\section{Competing interests}

The authors declare that they have no competing interests.

\section{Author details}

${ }^{1}$ Neurology Clinic, University Clinical Center of Serbia, Dr. Subotic Street 6, Belgrade 11000, Serbia. ${ }^{2}$ Clinic of Neurology and Psychiatry for Children and Youth, Dr. Subotic Street 6a, Belgrade 11000, Serbia. ${ }^{3}$ Medical Faculty, University of Belgrade, Dr. Subotic Street 8, Belgrade 11000, Serbia. ${ }^{4}$ Clinic of Nephrology, University Clinical Center of Serbia, Pasterova Street 2, Belgrade 11000, Serbia.

Received: 8 April 2021 Accepted: 26 May 2021

Published online: 15 June 2021

References

1. Gioffredi A, Maritati F, Oliva E, Buzio C. Eosinophilic granulomatosis with polyangiitis: an overview. Front Immunol. 2014:5:549.

2. Mouthon L, Dunogue B, Guillevin L. Diagnosis and classification of eosinophilic granulomatosis with polyangiitis (formerly named ChurgStrauss syndrome). J Autoimmun. 2014;48-49:99-103.

3. Guillevin L, Lhote F, Gayraud M, Cohen P, Jarrousse B, Lortholary O, et al. Prognostic factors in polyarteritis nodosa and Churg-Strauss syndrome. A prospective study in 342 patients. Medicine (Baltimore). 1996;75(1):17-28. https://doi.org/10.1097/00005792-199601000-00003.

4. André R, Cottin V, Saraux JL, Blaison G, Bienvenu B, Cathebras P, et al. Central nervous system involvement in eosinophilic granulomatosis with polyangiitis (Churg-Strauss): report of 26 patients and review of the literature. Autoimmun Rev. 2017;16(9):963-9. https://doi.org/10.1016/j.a utrev.2017.07.007.

5. Jennette JC, Falk RJ, Andrassy K, Bacon PA, Churg J, Gross WL, et al. Nomenclature of systemic vasculitides. Proposal of an international consensus conference. Arthritis Rheum. 1994;37(2):187-92. https://doi.org/1 0.1002 /art.1780370206.

6. Groh M, Pagnoux C, Baldini C, Bel E, Bottero P, Cottin V, et al. Eosinophilic granulomatosis with polyangiitis (Churg-Strauss) (EGPA) Consensus Task Force recommendations for evaluation and management. Eur J Intern Med. 2015;26(7):545-53. https://doi.org/10.1016/j.ejim.2015.04.022.

7. Comarmond C, Pagnoux C, Khellaf M, Cordier JF, Hamidou M, Viallard JF, et al. Eosinophilic granulomatosis with polyangiitis (Churg-Strauss): clinical characteristics and long-term followup of the 383 patients enrolled in the French Vasculitis Study Group cohort. Arthritis Rheum. 2013;65(1):270-81. https://doi.org/10.1002/art.37721.

8. Ghaeni L, Siebert E, Ostendorf F, Endres M, Reuter U. Multiple cerebral infarctions in a patient with Churg-Strauss syndrome. J Neurol. 2010;257(4): 678-80. https://doi.org/10.1007/s00415-009-5439-1.

9. Specks U, Merkel PA, Seo P, Spiera R, Langford CA, Hoffman GS, et al. Efficacy of remission-induction regimens for ANCA-associated vasculitis. N Engl J Med. 2013;369(5):417-27. https://doi.org/10.1056/NEJMoa1213277.

10. Grayson PC, Monach PA, Pagnoux C, Cuthbertson D, Carette S, Hoffman GS, et al. Value of commonly measured laboratory tests as biomarkers of disease activity and predictors of relapse in eosinophilic granulomatosis with polyangiitis. Rheumatology (Oxford). 2015;54(8):1351-9. https://doi. org/10.1093/rheumatology/keu427.

11. Ames PR, Margaglione M, Mackie S, Alves JD. Eosinophilia and thrombophilia in Churg Strauss syndrome: a clinical and pathogenetic overview. Clin Appl Thromb Hemost. 2010;16(6):628-36. https://doi.org/1 $0.1177 / 1076029609348647$.

\section{Publisher's Note}

Springer Nature remains neutral with regard to jurisdictional claims in published maps and institutional affiliations. 